\title{
ILCEA
}

Revue de l'Institut des langues et cultures

d'Europe, Amérique, Afrique, Asie et Australie

$30 \mid 2018$

Création culturelle et territoires : de l'histoire au mythe, du réel à l'utopie

\section{Imaginaire national et territoire : la construction nationale marocaine après l'indépendance}

National Imagination and Territory: Nation-Building in Morocco after the Independence

Eva Cantat

\section{OpenEdition}

Journals

Édition électronique

URL : https://journals.openedition.org/ilcea/4468

DOI : $10.4000 /$ ilcea.4468

ISSN : 2101-0609

Éditeur

UGA Éditions/Université Grenoble Alpes

Édition imprimée

ISBN : 978-2-37747-033-4

ISSN : $1639-6073$

\section{Référence électronique}

Eva Cantat, «Imaginaire national et territoire : la construction nationale marocaine après

l'indépendance », ILCEA [En ligne], 30 | 2018, mis en ligne le 31 janvier 2018, consulté le 21 septembre 2021. URL : http://journals.openedition.org/ilcea/4468 ; DOI : https://doi.org/10.4000/ilcea.4468

Ce document a été généré automatiquement le 21 septembre 2021.

(C) ILCEA 


\title{
Imaginaire national et territoire : la construction nationale marocaine après l'indépendance
}

\author{
National Imagination and Territory: Nation-Building in Morocco after the \\ Independence
}

Eva Cantat

1 L'histoire du Maroc indépendant présente la particularité de commencer par un processus de réunification territoriale et administrative. En effet, suite à la conférence d'Algésiras (1906), puis au traité de Fès (1912), le Maroc avait été scindé en trois zones pendant près d'un demi-siècle: un protectorat français, un protectorat espagnol, ainsi qu'un espace sous administration internationale (la zone internationale de Tanger). C'est sur le socle de ces divisions qu'il a fallu bâtir, à partir du printemps 1956, un nouvel État indépendant dont la souveraineté allait devoir être reconnue aussi bien à l'intérieur qu'à l'extérieur du pays. Cette édification d'un État centralisé sous l'égide des Alaouites - une dynastie qui donna au Maroc tous ses Sultans à partir de la seconde moitié du XVII ${ }^{\mathrm{e}}$ siècle - ne pouvait être menée à bien que si elle était accompagnée d'un véritable processus de construction nationale. La "nation", entendue d'après la désormais classique définition de Bénédicte Anderson comme une "communauté politique imaginaire, et imaginée comme intrinsèquement limitée et souveraine » $(1996: 19)$ repose avant toute chose sur la notion de communauté et sur un socle de « valeurs politiques et culturelles " (Lacoste, 2010 : 412) que les membres d'une société se donnent en partage ; ces valeurs leurs permettent également de se distancier de l'altérité. Or, cette notion de communauté concorde peu avec ce qu'était le Maroc du milieu des années 1950. Il était en effet particulièrement difficile de générer une identification nationale au sein de cette société, car elle était profondément divisée. Les éléments qui avait été définis à partir des années 1930 par les mouvements nationalistes marocains comme les fondements de l'identité marocaine - islam, langue arabe et monarchie alaouite - (Velasco de Castro, 2013) ne suffirent pas à sceller l'union nationale après l'indépendance, car le pays reposait sur une profonde complexité. 


\section{Une société fragmentée}

2 Comme mentionné précédemment, le morcellement de la société marocaine nouvellement indépendante provenait notamment de la division opérée par l'administration coloniale. Pendant la période des protectorats, les trois zones (espagnole, française, internationale) connurent un développement inégal en termes d'infrastructures ou encore d'éducation. Les dirigeants des protectorats espagnol et français optèrent également pour des choix politiques différents dans leurs espaces respectifs. Le soutien apporté par les Espagnols aux mouvements nationalistes marocains qui se développaient dans la zone française est à cet égard révélateur - malgré son caractère parfois ambivalent et superficiel (Velasco de Castro, 2016:353) -, tout comme l'appui donné par l'Espagne au sultan Mohamed Ben Youssef lorsque celui-ci fut destitué par les autorités françaises en 1953 au profit de son oncle Mohamed Ibn Arafa (Hernando de Larramendi Martínez, 1997 : 353). Ces choix politiques divergents étaient sous-tendus par de profondes rivalités franco-espagnoles.

3 Après l'indépendance, la réunification territoriale et administrative qui suivit ne résorba pas de fait les différences entre les deux anciens protectorats. L'histoire de la zone du Rif, une région située dans le nord-est du Maroc et comptant une importante population berbère, en est un exemple probant. Cette région, qui avait été sous protectorat espagnol, ne cessa de s'appauvrir avec le déclenchement de la guerre d'Algérie - notamment parce que les Rifains vivaient en partie de la culture des vignes dans l'Oranie voisine, rendues inaccessibles par l'éclatement du conflit. Le Rif se souleva en 1958 pour protester contre un sentiment d'abandon vis-à-vis de Rabat et contre l'absence de représentants rifains dans les instances de pouvoir marocaines (Ybarra Enríquez de la Orden, 1997; De Madariaga, 2010). Durement réprimé par les Forces armées royales, sous le commandement du jeune prince Mulay Hassan - qui devint trois ans plus tard le roi Hassan II -, ce soulèvement n'obtint pas l'effet escompté et aboutit au contraire à une très forte marginalisation du Rif au cours des décennies qui suivirent, comme en témoignent l'historien tétouanais Muhammad Ibn Azzuz Hakim (Morales Lezcano, 2002 : 259-260) et le leader nationaliste Abdelkhalek Torres (Dalle, 2004 : 359).

Ces divisions héritées de l'époque coloniale se superposaient, dans le Maroc du milieu des années 1950, à une fragmentation bien plus ancienne. Cette fragmentation ne se limitait pas à une dimension politique ou administrative puisqu'elle reposait également sur une importante diversité ethnique et culturelle. Le Maroc réunissait en effet des populations d'origines variées. Initialement peuplée par des ethnies berbères (dont l'implantation est antérieure au $\mathrm{v}^{\mathrm{e}}$ siècle), la côte ouest de l'Afrique du Nord connut ensuite l'arrivée de populations arabes lors des grandes conquêtes arabo-musulmanes qui suivirent la naissance de l'Islam. À ces deux principales ethnies s'ajoutait une population juive qui devint de plus en plus conséquente à la fin du Moyen Âge, du fait de l'arrivée des Séfarades chassés d'Espagne. Enfin, une population européenne d'origine coloniale vint compléter la diversité démographique de cet espace. Cette fragmentation ethnique fut d'ailleurs renforcée par le traitement différencié de la population berbère mis en place par l'administration coloniale de la zone française, comme en atteste notamment le dahir berbère de 1930 (Lafuente, 1984).

5 La superposition des caractéristiques ethniques des populations avec le découpage colonial donna lieu à une très grande diversité linguistique. Au milieu du xxe siècle, on 
trouvait au Maroc pas moins de six langues en contact (Benzakour, Gaadi \& Queffélec, 2000). Coexistaient l'amazighe, une langue comprenant trois variantes dialectales: le tarifite, le tamazighte et le tachelhite (Boukous, 1995); l'arabe marocain ou darija, qui présentait également une diversité dialectale considérable (Youssi, 2004 [1991] ; Moscoso García, 2003); et le hassaniya, une variante éloignée de la darija, qui était parlée notamment dans le sud du Maroc (Benzakour, Gaadi \& Queffélec, 2000). Ces trois premières langues se trouvaient en situation de diglossie (Boukous, 1995) avec trois autres langues: l'arabe standard, une langue commune à l'ensemble du monde arabe principalement utilisée à l'écrit et dans des contextes formels (discours politiques, média, enseignement), ainsi que deux langues d'origine coloniale, le français et l'espagnol.

6 On entend par diglossie (Ferguson, 1959) une situation où, sur un même territoire, coexistent plusieurs langues et/ou dialectes qui ont des fonctions distinctes dans la société et qui font l'objet d'une évaluation symbolique de la part des locuteurs : dans le Maroc nouvellement indépendant, certaines étaient considérées comme supérieures (arabe standard, français et espagnol), tandis que les autres (amazighe ${ }^{1}$, darija ${ }^{2}$ et hassaniya) étaient perçues par les locuteurs comme inférieures (Boukous, 1995 ; Youssi, 2004 [1991]). L'emploi de ces différentes langues s'inscrivait dans une forte dichotomie entre l'oral et l'écrit, la vie privée et la vie professionnelle, et surtout, la connaissance et la maîtrise d'une ou plusieurs des langues considérées comme fortes témoignait d'un certain niveau d'éducation et d'une certaine appartenance socio-professionnelle. Les usages linguistiques recoupaient en effet des divisions sociales très importantes entre des élites urbaines ayant eu accès au système éducatif et des masses rurales pauvres et très peu alphabétisées. Après l'indépendance du Maroc, la compétition idéologique entre l'une des anciennes langues coloniales - le français - et l'arabe standard, mena à une politique d'arabisation; non seulement celle-ci ne donna pas lieu à l'homogénéisation linguistique recherchée, mais elle provoqua, comme l'ont démontré les travaux des sociolinguistes, une instrumentalisation du français au profit de la reproduction des élites (Boukous, 1995).

7 La diversité ethnique et linguistique propre à la société marocaine n'était pas une caractéristique anodine, car la composition ethnique et la langue sont des facteurs de cohésion nationale importants, comme en témoigne la construction des premiers Étatsnations européens. Ces derniers s'inspiraient en effet du modèle ethnique germanique ou du modèle civique français (dans lequel les questions linguistiques occupent une place importante). Mais au-delà de ces premières causes de morcellement, on peut se demander si le caractère tribal de la société marocaine ne constituait pas un facteur supplémentaire de division. Comme dans le reste du Maghreb et du monde arabe, loyauté à la famille, au clan et à la tribu primait sur la loyauté vis-à-vis des citoyens qui formaient partie de la même nation (Hédi Chérif, 2004). La structure tribale était d'ailleurs d'autant plus forte au Maroc que cette région d'Afrique du Nord n'avait pas été intégrée à l'Empire ottoman et n'avait donc pas été structurée par un modèle bureaucratique avant l'établissement des protectorats ${ }^{3}$. Le modèle de l'État-nation n'était donc pas une forme politique proche de l'organisation sociale traditionnelle de cet espace.

8 La construction nationale allait donc devoir s'accompagner d'une évolution de la structure politique du pays. Cela était d'autant plus compliqué que plusieurs projets politiques entrèrent rapidement en concurrence après l'indépendance. Avant la fin des protectorats, le sultan Mohamed Ben Youssef - qui devint en 1957 le roi Mohamed V avait été érigé, par les partis nationalistes, en icône du nationalisme marocain (Velasco 
de Castro, 2013). Il s'agissait donc dans un premier temps une figure plutôt consensuelle. Cependant, une fois l'indépendance obtenue, toutes les factions politiques de la société marocaine n'acceptèrent pas le choix de conserver une monarchie qui, bien que parlementaire, laissait une grande marge de pouvoir effectif au souverain. Le rôle de ce dernier était, en effet, plus de gouverner que de régner. La disparition brutale de Mohamed V en 1961 et l'arrivée au pouvoir de son jeune fils, Hassan II, vinrent nourrir une forte remise en question de la figure et des fonctions du roi. Cette contestation s'exprima souvent de manière violente, notamment lors des tentatives de coups d'État de 1971 et $1972^{4}$. La première décennie du règne de Hassan II fut le théâtre d'une grande instabilité pour la monarchie et de profondes divisions autour du projet politique du pays.

Cette brève, et par conséquent, incomplète esquisse des caractéristiques de la société marocaine du milieux $\mathrm{du} \mathrm{xx}^{\mathrm{e}}$ siècle permet de percevoir les difficultés que supposait la construction d'une unité nationale dans le Maroc des années qui suivirent l'indépendance. La diversité ethnique et culturelle évoquée est propre à la plupart des pays issus de la décolonisation, où elle a d'ailleurs donné lieu, dans de nombreux cas, à des conflits communautaires (dans les Balkans, en Afrique subsaharienne, ou encore au Moyen-Orient). Le Maroc a d'une certaine manière été capable d'unifier sa population de manière à limiter - sans toutefois éviter - l'émergence de conflits internes. La répression n'est sans doute pas étrangère à cela, mais elle n'a pas été le seul facteur en jeu.

10 Il est probable que l'histoire de la construction nationale marocaine ait reposé sur un important travail d'oubli : l'oubli de ce qui divisait, dispersait et fragmentait ce peuple marocain si pluriel et divers. Ce travail d'oubli fait d'ailleurs certainement partie intégrante de tous les processus de construction nationale puisqu'à la fin du XIX ${ }^{\mathrm{e}}$ siècle, se référant aux nouveaux États-nations européens et américains, Ernest Renan concluait déjà :

L'oubli, et je dirai même l'erreur historique, sont un facteur essentiel de la formation d'une nation [...]. L'essence d'une nation est que tous les individus aient beaucoup de choses en commun, et aussi que tous aient oublié bien des choses. (Renan, 2012 [1882] : 7-9)

11 Pour que ce processus d'oubli se produise au Maroc, il a fallu trouver une cause commune autour de laquelle la population allait pouvoir se fédérer : le territoire a joué à cet égard un rôle déterminant.

\section{Territoire et construction nationale}

L'indépendance du Maroc commença en mars 1956 avec la fin du protectorat de la zone française. La décolonisation du Maroc par la France fut rapide et peu conflictuelle, car le gouvernement français préférait concentrer ses forces dans le conflit qui était alors en cours avec l'Algérie. Le processus se poursuivit en avril 1956, date à laquelle l'Espagne dut mettre un terme à son protectorat de manière assez précipitée. Dans ce second cas, la décolonisation se déroula de manière progressive et elle s'avéra difficile; l'Espagne ne restitua dans un premier temps au Maroc que la zone nord du protectorat, laissant en suspens le devenir d'autres territoires aux statuts et aux histoires hétérogènes comme Tarfaya, l'enclave d'Ifni, le Sahara espagnol, Ceuta, Melilla, les îles Zaffarines, les îles d'Al Hoceïma et le rocher de Vélez de la Gomera. La décolonisation de la zone espagnole donna 
lieu à plusieurs décennies de conflits dont les principaux enjeux étaient militaires (retrait des troupes espagnoles), économiques (question de la pêche), mais surtout territoriaux - un enjeu qui recoupe les deux autres puisque la maitrise du territoire implique des avantages stratégiques ainsi que le contrôle des ressources.

La diplomatie franquiste percevait ces conflits territoriaux avec le Maroc comme une rébellion postcoloniale, c'est-à-dire, comme une forme de rejet absolu de l'ancienne puissance occupante par l'État nouvellement indépendant (Pardo, 2006). Mais si les tensions étaient aussi fortes avec l'Espagne, c'est aussi parce que les enjeux territoriaux étaient perçus comme particulièrement importants par le Maroc. En effet, dans l'imaginaire nationaliste marocain, le territoire occupa très tôt une place prépondérante : en témoigne la théorie du Grand Maroc présentée et défendue quelques années avant l'indépendance par le leader istiqlalien Allal El Fassi (El Fassi, 1948). En juillet 1956, ce dernier rendit publique dans le journal de l'Istiqlal, Al Alam, une carte qui devint progressivement une référence dans l'imaginaire territorial marocain. D'après ce document, le Grand Maroc devait recouvrir l'ensemble du territoire administré par les Français et les Espagnols, mais aussi toute la partie ouest du désert algérien, le nord du Mali ainsi que l'intégralité de la Mauritanie. Cet espace était défini par les nationalistes marocains comme les limites historiques du Maroc (Lazrak, 1974).

Témoigne également de ces revendications territoriales une lettre que le roi Mohamed V adressa à Franco en 1960. Il y annonçait sa volonté de faire table rase du découpage territorial colonial :

Nos deux peuples doivent résoudre leurs différends dans l'intérêt de leurs générations futures [...] en fondant leurs échanges sur les bases de l'égalité et du respect mutuel, en écartant toutes les idées qui ne concordent désormais plus avec notre temps, et en renonçant à tous les privilèges et droits concédés en vertu d'accords archaïques dont la concertation avait eu lieu dans des circonstances exceptionnelles. (Palacios, 2005 : 399)

Il apparaît clairement que le Maroc indépendant n'entendait pas renoncer à une bonne partie du Grand Maroc: la région de Tarfaya fut récupérée en 1958; la frontière avec l'Algérie fut contestée en 1963, ce qui donna lieu à un conflit armé connu sous le nom de guerre des Sables ; l'enclave de Sidi Ifni fut récupérée en 1969 suite à des négociations avec l'Espagne ; la Mauritanie ne fut reconnue par le Maroc qu'en 1970 (c'est-à-dire, dix ans après son indépendance); et enfin, le Sahara occidental fut rattaché au Maroc en 1975 suite à la Marche verte et aux accords de Madrid qui en découlèrent. Quelques mois après ces accords et plus de vingt-cinq ans après la lettre que Mohamed $\mathrm{V}$ avait adressée à Franco, Hassan II affirmait encore :

Je suis heureux que le bon droit ait finalement et pacifiquement prévalu en ce qui concerne notre Sahara. J'ai bonne espérance qu'un jour on reconnaîtra de même que Sebta, Melilla, les îles du Rif sont territoires marocains. (El Alaoui, 1976: 88)

Une grande partie des spécialistes du Maroc s'accordent à penser que la thèse du Grand Maroc bénéficiait d'une adhésion profonde et sincère dans la plupart des couches de la société marocaine, et tout particulièrement auprès des classes populaires qui voyaient en elle l'espoir d'un retour à une prospérité économique et politique :

Ce sur quoi il convient d'insister, c'est l'unanimité de la population marocaine, à travers ses composantes sociales, syndicales et politiques, sur la marocanité du Sahara occidental. Au niveau des masses populaires, l'adhésion profonde et sincère procède très certainement d'une représentation de la restauration du Grand Maroc. La reconquête des territoires jadis sous maîtrise marocaine devrait permettre le 
rétablissement d'un âge d'or économique et politique. Les richesses supposées du Sahara entraîneraient une amélioration du niveau de vie de l'ensemble de la population; l'accroissement territorial placerait le Maroc dans une situation prépondérante quant à la construction d'un Maghreb uni. (Bontemps, 1984 : 107)

Cependant, on ignorait à quel point la région de Bucraa était riche en phosphates au moment où cette thèse a commencé à se développer et la revendication du Sahara occidental par le Maroc a été antérieure d'une dizaine d'années à la découverte des ressources naturelles dont regorge ce territoire (Damis, 1983). C'est donc bien l'imaginaire symbolique, plus que le profit économique, qui portait initialement ce projet.

Il existe effectivement un lien étroit entre imaginaire et territoire. Le géographe français Roger Brunet souligne la fascination que les lieux produisent dans l'imaginaire individuel et social, que ces lieux soient connus ou inconnus, voire même fictifs - comme l'Eldorado ou le pays de Cocagne - (Brunet, 2001). La sociologue catalane Maria Montserrat Guibernau insiste sur ce caractère imaginaire et fantasmé du territoire : il arrive que des peuples soient prêts à se sacrifier pour des territoires dont ils revendiquent la souveraineté alors qu'ils ne les connaissent pas et ne les visiteront jamais (Guibernau, 2007). Or, dans le cas qui nous intéresse, très peu de Marocains et encore moins d'Espagnols seront amenés à se rendre dans les territoires revendiqués, et plus particulièrement, au Sahara occidental.

Cet auteur évoque également l'importance des symboles et des rituels propres à chaque nation (Guibernau, 2007), et l'on peut dire que la carte du Grand Maroc, au même titre que la fête du Trône ${ }^{5}$, fait partie de ces constituants symboliques de la nation marocaine. Guibernau s'accorde ici avec la thèse de Benedict Anderson, qui considère que l'élaboration d'une mythologie nationale constitue l'un des ingrédients essentiels de la construction de la nation (Anderson, 1996). À l'échelle du Maroc, la mythologie nationale comprend non seulement la carte présentée par El Fassi, mais aussi la Marche verte de 1975 qui, par l'utilisation des civils - certes, encadrés par l'armée - comme bouclier humain face aux troupes franquistes, a constitué une forme d'épopée collective dont l'acteur central était le peuple marocain - sans distinctions d'aucune sorte. L'intellectuel marocain Abdallah Laroui, cité par Ignace Dalle, l'exprime en ces termes:

La Marche verte ne fut pas seulement un acte politique : elle fut autre chose. Quoi donc? Il n'est pas facile de trouver le qualificatif adéquat. Pour faire bref, utilisons une formule bien familière aux essayistes français depuis Charles Péguy, et disons qu'elle fut aussi un acte mystique. (Dalle, $2004: 428$ )

C'est aussi le caractère mystique et mythique de la marche qui fut mis en avant dans le récit qu'en dressa le monarque Hassan II quelques mois après les événements :

Ils [les marcheurs] pénétrèrent massivement, bien qu'en ordre parfait, sur le territoire en litige et nul ne peut dire exactement la distance qu'ils y parcoururent jusqu'au 9 novembre: de vingt à trente kilomètres environ. L'ardeur de leur foi était si grande qu'ils fussent allés jusqu'au bout du monde : ils avaient gagné et l'ignoraient encore. (El Alaoui, 1976 : 180)

21 Notons également que l'opposition à un ennemi commun permet de réunir masses et élites dans une même dynamique (Guibernau, 2007). Et dans une plus ample optique, « il n'y a pas de moyen plus efficace de lier ensemble des sections disparates d'un peuple agité que de les unir contre des étrangers » (Hobsbawn, 1992). La figure de l'ennemi commun a effectivement la capacité de fédérer les peuples divisés; ce phénomène est d'autant plus fort lorsque l'ennemi semble s'attaquer à l'intégrité d'un territoire considéré comme national. Le géopolitologue Yves Lacoste perçoit à ce titre la nation 
comme une représentation géopolitique, c'est-à-dire comme intrinsèquement liée au territoire. D'après lui,

[la nation] implique des rivalités de pouvoir sur du territoire, car il n'y a pas de nation sans volonté d'indépendance contre de possibles adversaires et parce qu'il n'y a pas de nation sans territoire et sans limites territoriales qui ont été plus ou moins contestées par des nations rivales. (Lacoste, $2010: 412$ ) aurait donc tendance à générer une cohésion intérieure, qui permettrait ensuite d'assurer la pérennité du système. C'est ce que Jean-Frédéric Morin résume en ces termes : «C'est l'insécurité de la nation qui fonde la sécurité de l'État. » (2013 : 110)

Pour en revenir au Maroc, il semble clair que par ces conflits territoriaux, les Marocains ont trouvé des ennemis communs. L'Algérie tout d'abord, pays voisin avec lequel les tensions ont été récurrentes à partir de l'indépendance de celui-ci. Le point culminant fut sans doute le conflit armé de 1963, dont les principales causes étaient justement territoriales et remettaient en cause le tracé des frontières établies à l'époque coloniale. L'Algérie socialiste de Ben Bella puis de Boumédiène reposait sur un système politique opposé au modèle marocain (Hernando de Larramendi Martínez, 1997: 328) et représentait une menace pour la conservatrice monarchie alaouite; cette situation aboutit à une reproduction à l'échelle du Maghreb de la bipolarité internationale de la guerre froide (Berramdane, 1987). Dans le contexte de la guerre des Sables qui opposa les deux pays en 1963, l'Algérie bénéficia de soutiens internationaux - comme en témoigne l'appui fourni par Cuba (Gleijeses, 1996) et par la République arabe unie ${ }^{6}$ (Wild, 1966 ; Torres García, 2012). L'intervention de ces derniers rendit difficile une véritable remise en question de la frontière algéro-marocaine et le dénouement de cette courte guerre fut donc scellé par un maintien des frontières antérieures au conflit.

Le Maroc trouva en revanche dans l'Espagne un ennemi commun bien plus commode. Tout d'abord parce qu'il s'agissait d'une ancienne puissance coloniale et que les tensions pouvaient donc être présentées à l'opinion publique sous le prisme de la décolonisation, un angle de vue tout à fait propice à susciter l'adhésion populaire dans le contexte maghrébin des années 1960. Il n'en était pas de même avec l'Algérie, avec laquelle le Maroc entretenait, selon le discours officiel, une certaine solidarité face aux anciennes puissances coloniales malgré les tensions récurrentes entre les deux pays. Par ailleurs, l'Espagne n'avait pas tissé un réseau d'intérêts économiques forts et pérennes avec le Maroc, comme avait su l'établir la France. Enfin, l'Espagne était alors un pays marginalisé sur la scène internationale puisqu'elle en avait été mise au ban après la Seconde Guerre mondiale du fait de sa sympathie pour l'Allemagne nazie et l'Italie fasciste. Malgré la rupture de l'isolement espagnol en 1953 grâce à la signature d'un traité avec les ÉtatsUnis, ces derniers n'accordèrent que peu de soutien à l'Espagne dans les conflits hispanomarocains, car ils préféraient s'assurer la fidélité et la coopération militaire du Maroc dans le contexte de polarisation que connaissait alors le Maghreb. Dans cette période de guerre froide, le gouvernement franquiste, qui avait fait de l'anticommunisme son étendard, ne risquait quant à lui en aucune manière de se rapprocher du bloc soviétique.

Cette Espagne faible et isolée représentait donc un ennemi convenable pour le Maroc, et c'est sur ce terrain propice que se sont développés de multiples conflits territoriaux entre les deux pays. Un dernier point mérite cependant d'être évoqué : si le territoire a joué une fonction symbolique aussi importante dans le Maroc postcolonial, c'est certes parce qu'il 
a contribué à l'édification d'une unité nationale dans ce pays morcelé, mais aussi parce qu'il a considérablement servi les desseins de la monarchie alaouite.

\section{Territoire et monarchie}

Comme nous l'avons mentionné précédemment, les premières années du règne de Hassan II furent très difficiles. La remise en question de son statut et de ses fonctions atteignit son paroxysme au début des années 1970, lorsqu'il échappa de peu à deux tentatives successives de coups d'État militaires. Par conséquent, Hassan II comprit rapidement l'importance des enjeux symboliques dont il allait avoir besoin pour rétablir le prestige de la monarchie et assurer sa légitimité comme souverain alaouite. Il s'appuya en premier lieu sur la religion, dès son intronisation (El Houdaïgui, 2003) : le statut d' Amir-al-muminin (Commandeur des croyants) qu'il détenait parce qu'il était un lointain descendant du prophète Mahomet par la branche de son gendre Ali, lui permettait en effet tout à la fois de se poser en gardien de la tradition musulmane et de limiter les contestations islamistes. Cette très haute fonction religieuse lui permettait surtout de bénéficier d'une légitimité religieuse, à défaut d'une légitimité démocratique.

Au cours des années 1960, le jeune roi s'engagea progressivement dans un autre champ très porteur sur le plan symbolique: celui de la politique extérieure. Dans sa gestion politique, Hassan II verrouilla progressivement les affaires étrangères jusqu'à en faire un domaine réservé. Cette prérogative fut renforcée par un ancrage constitutionnel, comme en témoigne l'article 19 de la première Constitution du Maroc indépendant :

Le Roi, «Amir Al Mouminine » (commandeur des croyants), symbole de l'unité de la nation, garant de la pérennité et de la continuité de l'État, veille au respect de l'Islam et de la Constitution. Il est le protecteur des droits et libertés des citoyens, groupes sociaux et collectivités. Il garantit l'indépendance de la nation et l'intégrité territoriale du royaume dans ses frontières authentiques. (Constitution, 1962)

Alors que la politique extérieure du royaume était initialement, après l'indépendance, sous gouvernance partagée avec les partis du Mouvement national ${ }^{7}$ - qui occupaient portefeuilles et sièges au ministère des Affaires étrangères ainsi qu'à l'Assemblée nationale consultative - elle devint la chasse gardée du monarque à partir de l'État d'exception de 1965 (Hernando de Larramendi Martínez, 1997).

Du fait de l'État d'exception, les partis du Mouvement national se retrouvèrent brutalement exclus du gouvernement et passèrent donc dans l'opposition; or, c'étaient eux qui étaient jusqu'alors porteurs de la mythologie nationale marocaine, qu'il s'agisse d'événements comme la fête du Trône ou de thèses historiques comme celle du Grand Maroc. Hassan II, qui ne pouvait prendre le risque de laisser entre les mains de l'opposition un discours aussi mobilisateur, se l'appropria donc progressivement (Hernando de Larramendi Martínez, 1997) : dans une première période (1965-1973), il reprit à son compte le discours nationaliste et territorial de l'opposition tout en excluant celle-ci du jeu politique extérieur; dans un second temps (à partir de 1974), il réintégra sous sa coupe les partis d'opposition afin de faire campagne en faveur de ses propres décisions en matière de politique extérieure, en vue notamment, de la préparation de la Marche verte.

De cette manière, non seulement le discours nationaliste de l'opposition perdit son caractère contestataire - puisqu'il était assumé et revendiqué par la monarchie -, mais il devint même un discours de ralliement derrière la figure du souverain. Dans le contexte 
de très forte instabilité politique qui régnait au Maroc au début des années 1970, la Marche verte permit de créer un véritable phénomène de ralliement au drapeau ${ }^{8}$. Cette situation est habituellement temporaire, mais elle a constitué au Maroc l'impulsion d'une dynamique plus longue, parce que l'aura créée sur le plan national s'est répercutée sur la scène internationale, en sonnant le coup final du renversement du rapport de forces entre l'Espagne et le Maroc (Ybarra Enríquez de la Orden, $2006: 236$ ). Suite à la Marche verte de novembre 1975, l'Espagne a perdu beaucoup de prestige sur la scène internationale alors que le Maroc a renfoncé son statut de protégé des États-Unis et de la France, tout en devenant par la même occasion un grand leader à l'échelle du monde arabe. Au niveau national Hassan II s'est posé en réunificateur du royaume alaouite, associant ainsi sa dynastie à l'heureux aboutissement de la mythologie territoriale élaborée par les nationalistes.

31 Sans perdre de vue ou minimiser les enjeux stratégiques et économiques de la conquête marocaine du Sahara occidental, il semble nécessaire de souligner que la Marche verte et le rattachement d'une partie du Sahara occidental au Maroc (suite aux accords de Madrid) ont permis d'assurer la pérennité de la monarchie alaouite à un moment où celleci était très fortement remise en question.

L'histoire du Maroc nouvellement indépendant illustre de manière très éclairante la réflexion portée par cet ouvrage sur l'imaginaire et le territoire, ici dans le cadre de la construction nationale des pays issus de la décolonisation. Elle constitue pour l'historien et le politologue un intéressant laboratoire où observer les enjeux symboliques attachés au territoire, la capacité de celui-ci à unifier au-delà des clivages sociaux, le rôle du conflit externe dans la construction nationale, et surtout, l'articulation constante entre les enjeux de politique extérieure et ceux de politique intérieure.

\section{BIBLIOGRAPHIE}

ANDERSON Benedict (1996), L'imaginaire national. Essai sur l'origine et l'essor du nationalisme, Paris : La Découverte.

BENZAKOUR Fouzia, GAADI Driss \& QUEFFÉLEC Ambroise (2000), Le français au Maroc : lexique et contacts de langues, Louvain : De Boeck Supérieur.

BERRAMDANE Abdelkhaleq (1987), Le Maroc et l'occident : 1800-1974, Paris : Karthala Éditions. BontEMs Claude (1984), La guerre du Sahara occidental, Paris : Presses universitaires de France. BOUHJAR Aïcha (2012), « De la langue du terroir à une langue au pouvoir : officialisation de la langue amazighe (berbère) au Maroc », Lengas. Revue de sociolinguistique, 71, 29-40.

Boukous Ahmed (1995), Société, langues et cultures au Maroc : enjeux symboliques, Rabat : Faculté des lettres et des sciences humaines.

BRUNET Roger (2001), Le déchiffrement du monde : théorie et pratique de la géographie, Paris : Belin. 
Constitution du 7 décembre 1962, Maroc, en ligne sur Digithèque de matériaux juridiques et politiques, Université de Perpignan, <http://mjp.univ-perp.fr/constit/ma1962.htm> (27 juin 2017).

DALLE Ignace (2004), Les trois rois : la monarchie marocaine de l'indépendance à nos jours, Paris : Fayard.

DALLE Ignace (2007), Maroc : histoire, société, culture, Paris : La Découverte.

DAMIS John James (1983), Conflict in Northwest Africa: The Western Sahara Dispute, Stanford, CA : Hoover Institution Press.

De MADARIAGa María Rosa (2010), «El Rif y el poder central: una perspectiva histórica », Revista de Estudios Internacionales Mediterráneos, 9, 1-9.

El Alaoui Hassan (1976), Le défi, Paris : Albin Michel.

EL FASSI Allal (1948), Los movimientos de independencia en el Mogreb Árabe, Marruecos : Comisión de Cultura nacionalista del partido Al Istiqlal (Ouvrage original publié sous le titre Al-harakat alistiqlalia fi-al-magrib al-arabi, Le Caire : Al Risala).

EL HOUDAïGUI Rachid (2003), La politique étrangère sous le règne de Hassan II : acteurs, enjeux et processus décisionnels, Paris : L'Harmattan.

FERGUSON Charles A. (1959), « Diglossia », Word, 15, 325-340.

GELLNER Ernest (1969), Saints of the Atlas, Londres : Weidenfeld \& Nicolson.

GLeIJESES Piero (1996), « Cuba's First Venture in Africa: Algeria, 1961-1965 », Journal of Latin American Studies, 28, 159-195.

GUIBERNAU Montserrat (2007), The Identity of Nations, New York : John Wiley \& Sons.

HÉDI CHÉRIF Mohamed (2004), «L'empreinte des appartenances communautaires sur les sociétés », C. Lacoste-Dujardin \& Y. Lacoste (dir.), Maghreb : peuples et civilisations, Paris : La Découverte, 99-107.

HERNANDO DE LARRAMENDi MARTínez Miguel (1997), La política exterior de Marruecos, Madrid: Fundación MAPFRE.

HoBSBAWN Eric (1992), Nations et nationalismes depuis 1780, Paris : Gallimard.

LACOSTE Yves (1998), Vive la nation : destin d'une idée géopolitique, Paris : Fayard.

LACOSTE Yves (2010), La question post-coloniale : une analyse géopolitique, Paris : Fayard.

LAFUENTE Gilles (1984), « Dossier marocain sur le dahir berbère de 1930 », Revue de l'Occident musulman et de la Méditerranée, 1, 83-116.

LAZRAK Rachid (1974), Le contentieux territorial entre le Maroc et l'Espagne, Casablanca : Dar el Kitab. MORALES LEZCANO Víctor (2002), Diálogos ribereños: conversaciones con miembros de la elite marroquí, Madrid : UNED (Universidad Nacional de Educación a Distancia).

MORIN Jean-Frédéric (2013), La politique étrangère : théories, méthodes et références, Paris : Armand Colin.

Moscoso GARcíA Francisco (2003), « Situación lingüística en Marruecos: árabe marroquí, bereber, árabe estándar, lenguas europeas ", Al-Andalus Magreb, 10, 157-186.

PALAcios Jesús (2005), Las cartas de Franco, Madrid : La Esfera de los Libros. 
PARDo SANZ Rosa María (2006), « Una relación envenenada: España-Marruecos (1956-1969) », A. Mateos López \& A. Herrerin López (dir.), La España del presente: de la dictadura a la democracia, Madrid : Asociación de historiadores del Presente, 199-222.

Renan Ernest (2012), Qu'est-ce qu'une nation? [1882], Paris : République des Lettres.

TORRES GARCíA Ana (2012), La Guerra de las Arenas. Conflicto entre Marruecos y Argelia durante la Guerra Fría (1963), Barcelone : Bellaterra.

VELASCO DE CASTRO Rocío (2013), « La monarquía alauí, símbolo identitario de la nación marroquí: legitimidad histórica e instrumentalización política », Diacronie. Studi di Storia Contemporanea, 16 (4), 1-17.

Velasco De CASTRo Rocío (2016), « Políticas de sustitución, Guerra Fría y descolonización: la paradoja del Protectorado Español en Marruecos », C. Navajas Zubeldía \& D. Iturriaga Barco (dir.), Actas del V Congreso Internacional de Historia de Nuestro Tiempo, Logroño : Universidad de la Rioja, 349-362.

WILD Patricia Berko (1966), « The Organization of African Unity and the Algerian-Moroccan Border Conflict: A Study of New Machinery for Peacekeeping and for the Peaceful Settlement of Disputes among African States ", International Organization, 20, 18-36.

YBARRA ENRÍQUez DE LA ORDEN María Concepción (1997), « La rebelión del Rif (1958-1959) », Espacio Tiempo y Forma. Serie V, Historia Contemporánea, 10, 333-347.

YBARRA ENRÍQUeZ DE LA ORDEN María Concepción (2006), « Relaciones hispano-marroquíes en los inicios del reinado de Hassan II », A. Mateos López \& A. Herrerin López (dir.), La España del presente: de la dictadura a la democracia, Madrid : Asociación de historiadores del Presente, 223-236.

Youssi Abederrahim (2004), « Un trilinguisme complexe », C. Lacoste-Dujardin \& Y. Lacoste (dir.), Maghreb : peuples et civilisations [1991], Paris : La Découverte, 99-107.

\section{NOTES}

1. La représentation symbolique de l'amazighe au Maroc a connu une évolution au cours des décennies postérieures. En témoigne le fait qu'elle ait été reconnue comme l'une des langues officielles du royaume dans la Constitution de 2011 (Bouhjar, 2012).

2. L'arabe dialectal représentait en revanche une variété haute dans la relation diglossique qu'il entretenait avec l'amazighe (Boukous, 1995 : 57).

3. L'on peut se référer à ce sujet aux recherches d'Ernest Gellner, qui a mené des travaux en anthropologie sociale sur la société tribale dans le Maroc des années 1960 (Gellner, 1969).

4. Le coup d'État de Skhirat en juillet 1971 et le coup d'État des aviateurs en août 1972.

5. Il s'agit d'une fête annuelle instaurée par les milieux nationalistes marocains en 1933 et devenue officielle l'année suivante. Elle commémore l'anniversaire de l'intronisation du souverain en place.

6. Il s'agit en réalité de l'Égypte, qui a porté le nom de République arabe unie pendant plusieurs années après la dissolution de celle-ci en 1961.

7. Partis issus de la lutte anticoloniale comme l'Istiqlal ou le Parti démocratique de l'indépendance.

8. Mécanisme par lequel on observe une cohésion très forte de toutes les franges de la société autour du chef de l'État et/ou du chef du gouvernement en exercice lorsqu'un pays est projeté sur le devant de la scène internationale par un événement tragique ou au contraire valorisant. 


\section{RÉSUMÉS}

Au terme de la période coloniale, la société marocaine se trouvait profondément divisée : non seulement elle avait été répartie entre trois administrations coloniales pendant plusieurs décennies, mais elle comportait surtout une grande diversité culturelle et linguistique, souvent renforcée par de profonds contrastes socio-économiques entre les zones rurales et urbaines. À cette fragmentation sociale et culturelle s'ajoutait la diversité des projets présents sur la scène politique, dont certains n'étaient pas favorables au gouvernement effectif de la monarchie alaouite. Le pouvoir du souverain fut de ce fait constamment remis en question dans les deux décennies qui suivirent l'indépendance. Dans un tel contexte, on peut se demander si la récupération par le roi Hassan II du discours nationaliste portant sur les questions territoriales n'aurait pas été - outre une occasion de soustraire de l'influence à l'un de ses principaux opposants politiques (le parti de l'Istiqlal) - un outil pour mobiliser l'ensemble de cette société si diverse et si divisée vers un objectif national commun. Et, de cette même façon, d'utiliser le développement de ce sentiment national afin de préserver l'ordre monarchique.

The end of the colonial era left Moroccan society deeply divided: it had been ruled by three different colonial administrations, it consisted of a large cultural and linguistic diversity, and was characterized by enormous contrasts between the urban world and the rural areas. Added to this social and cultural fragmentation was the plurality of the existing political projects which were not all in favor of the effective Government of the Alawi monarchy-whose power would later be questioned as demonstrated by the Rif rebellion in the late fifties as well as the coup attempts of 1971 and 1972. In such a context, one may wonder whether King Hassan II used the restoration of the Nationalist discourse on the outstanding territorial issues with Spain not only as an opportunity to reduce the influence of its main threat in the country's political sectors (the Istiqlal Party), but also as a pretext to mobilize the entirety of such a diverse and divided society on a common national goal, and, therefore, relate such sense of national identity to the preservation of the monarchical power.

\section{INDEX}

Keywords : Morocco, Spain, decolonization, territory, Nation, identity, Hassan II

Mots-clés : Maroc, Espagne, décolonisation, territoire, nation, identité, Hassan II

\section{AUTEUR}

\section{EVA CANTAT}

ILCEA4, Université Grenoble Alpes 